\title{
Anabases
}

ANABASES Traditions et réceptions de l'Antiquité

\section{Non solo pellegrini : Francesi a Roma nella prima età moderna. Qualche esempio e osservazione}

Irene Fosi

\section{(2) OpenEdition}

Journals

Edizione digitale

URL: http://journals.openedition.org/anabases/3149

DOI: 10.4000/anabases.3149

ISSN: 2256-9421

Editore

E.R.A.S.M.E.

\section{Edizione cartacea}

Data di pubblicazione: 1 marzo 2007

Paginazione: 137-148

ISSN: 1774-4296

\section{Notizia bibliografica digitale}

Irene Fosi, « Non solo pellegrini : Francesi a Roma nella prima età moderna. Qualche esempio e osservazione », Anabases [Online], 5 | 2007, Messo online il 01 janvier 2012, consultato il 21 octobre 2019. URL : http://journals.openedition.org/anabases/3149 ; DOI : 10.4000/anabases.3149 
Anabases 5 (2007), p. 137-148

\section{Non solo pellegrini : Francesi a Roma nella prima età moderna. Qualche esempio e osservazione}

IRENE FosI

\section{Stranieri fra integrazione e identità}

Città di STRAnieri, sede della sola corte internazionale, Roma si presenta, all'inizio dell'età moderna, come un agglomerato di nationes che vivono insieme e separatamente. Città policroma e in continua crescita dal Quattrocento, per la stabile presenza della corte papale, Roma è sostenuta dal suo sviluppo politico, burocratico, artistico. L'articolazione duplice e strettamente interconnessa, fra corte e curia, aveva reso ineludibile la presenza ed il continuo aumento di forenses. Per la città del papa si può parlare di unitas multiplex, di unità composita che riconosceva diverse appartenenze, lealtà, identità non lesive o antagoniste, in maniera differente e a seconda dei tempi e delle circostanze, della pubblica quiete, dell'ordine pubblico, dell viver comune, e soprattutto dell'autorità del pontefice e dei suoi organismi di governo cittadino. Ma, è inutile sottolineare quanto questi concetti cambino di significato nei primi secoli dell'età moderna e, di conseguenza, come cambi anche la fisionomia delle comunità di stranieri che formavano questa multiforme società urbana. Se Roma condivide con altre città italiane ed europee i problemi posti dalla presenza di stranieri fra le sue mura, certo questa si connota di tratti peculiari per risiedere in una città con un duplice potere, capitale di una monarchia con un sovrano che, come è stato scritto, ha un corpo e due anime ${ }^{1}$. Dello straniero si guarderà in fatti anche l'anima, e sempre più dopo la rottura dell'unità cristiana in Europa. Nella policroma società cittadina dell'età moderna emergono infatti molte altre possibili forme di integrazione che permettevano a forestieri e

1 Il rinvio è, ovviamente, a P. PRODI, Il sovrano pontefice. Un corpo e due anime : la monarchia papale nella prima età moderna, Bologna, 1982. 
stranieri di inserirsi, assimilarsi e crescere, anche socialmente. La fisionomia di Roma, il dualismo corte/curia e città/municipio garantivano de iure e de facto diversi percorsi di integrazione ed assimilazione degli stranieri. E' poi necessario distinguere fra i protagonisti di un'immigrazione temporanea, stagionale o definitiva, fra la provenienza da zone circonvicine, da altri stati italiani, da Oltralpe. La condizione dello straniero di passaggio sarà infatti, per tutta la prima età moderna, connotabile con attributi negativi, difficile da controllare, facile bersaglio di autorità preposte a garantire l'ordine pubblico che coincideva con l'ordine morale e con l'ortodossia religiosa. A questa immagine negativa non si sottrarrà neppure il pellegrino che le autorità romane, attraverso più regolate forme di devozione e carità, come le confraternite, cercheranno di controllare e disciplinare, non solo nei tempi degli anni santi.

Poiché la società di antico regime percepiva e considerava il singolo solo come membro di una comunità, nella sua appartenza ad un milieu, anche quando l'immigrazione era temporanea e motivata dalla necessità di trovare un lavoro, diversamente si connotava la presenza del singolo o di una intera famiglia. Lo straniero, appena stabile diventava la sua presenza in città, entrava a far parte di una comunità, di una natio, elemento determinante di integrazione e, al tempo stesso, di separazione, almeno temporanea, rispetto alla società ospite. La condivisione di fattori identitari conferiva infatti stabilità, sicurezza, sfumando i caratteri negativi della condizione. Cambia, quindi, anche la percezione dello straniero da parte di chi era già inserito nel contesto cittadino : non più elemento eccentrico, liminale fra marginalità ed integrazione, fra norma e trasgressione, ma membro di una societas che risponde alle sue regole, sacralizzata dai suoi riti e cerimonie, con spazi ben individuabili nel contesto urbano : lo straniero diventava così controllabile per le autorità cittadine e papali sempre più attente. Decisivi per favorire l'integrazione, ma anche per conservare e difendere l'identità di appartenenza, diventavano le strategie matrimoniali segnate dall'endogamia, la lingua, la devozione ed il culto, la solidarietà nelle confraternite nazionali. L'inserimento di un individuo e della sua famiglia in una comunità nazionale aveva inoltre un significato pratico nel facilitare i rapporti con la comunità ospite e mantenere i legami con la città o la terra di origine. La necessità di entrare a far parte di una comunità già stabilmente inserita nella società romana, in questo caso, di riconoscersi in essa e riceverne legittimazione identitaria per non essere più stranieri rimane costante nel corso dell'età moderna, continuum di una prassi medievale, poco a poco superata da altri fattori di integrazione (o di esclusione) dello straniero nella società che lo aveva accolto. Dalla metà del Cinquecento si può senz'altro ascrivere la confessione religiosa fra i fattori identitari e gli elementi decisivi per l'integrazione.

\section{Conversioni religiose e propaganda politica}

La cesura della Riforma rese infatti fondamentale l'appartenenza confessionale come fattore di integrazione e, di conseguenza, la conversione religiosa. Certamente gli avve- 
nimenti bellici culminati nel Sacco di Roma del 1527 cambiarono la fisionomia di diverse nationes straniere a Roma, spingendo inoltre alla fuga verso altri stati italiani artigiani, artisti, letterati. Più tardi, poco più tardi, da Roma sarebbe fuggito anche chi era sospettato di eresia. L'identità religiosa, che presupponeva una rigida determinazione confessionale ed individuava nell'altro un nemico, dalla seconda metà del Cinquecento diventa per lo straniero un fattore essenziale di integrazione. Gli stranieri che vivevano a Roma, partecipi della sempre più complessa ed articolata realtà della corte pontificia e coinvolti nelle più diverse attività economiche della città - due entità strettamente correlate ed osmotiche - non gravitavano solo intorno alle numerose chiese nazionali. La cospicua presenza di nomi stranieri nei registri di diverse parrocchie romane è spia di un'integrazione non superficiale e occasionale che si rafforzava con la scansione dei più significativi riti di passaggio. Non sono neppure troppo rari i casi di stranieri che sceglievano padrini e madrine dei loro figli non fra i connazionali, ma piuttosto fra Romani, compagni di lavoro e vicini di casa, quasi per ricevere una sanzione ufficiale della avvenuta integrazione nella città ospite.

Il valore, simbolico e reale, della conversione intesa come cambiamento radicale di vita, materiale e spirituale, come assunzione volontaria e cosciente di una nuova identità, trovò dunque nella Roma del Sei e Settecento un terreno fertile per la sua utilizzazione, anche in senso politico ${ }^{2}$. Nella prima età moderna segnata da ostilità confessionali e terrore, il confine fra eresia ed ortodossia, ma anche fra diverse confessioni era, soprattutto per la gente comune, assai labile e sfumato e proprio a queste persone senza nome, prive di una identità politica, si deve anche guardare per comprendere il significato delle conversioni. Nascosti fra le torme di pellegrini che entravano in Roma non solo in occasione degli anni santi, ma che costantemente, con l'inizio della bella stagione, affluivano in città per inginocchiarsi alle tombe degli Apostoli gli stranieri erano dunque sempre presenti in città, pronti ad osservare talvolta con occhio malevolo, distorto dalla propaganda antiromana, tutto ciò che avveniva nella città del papa, così come ad irridere e denigrare costumi e abitudini dei suoi abitanti. A questi subdoli osservatori si doveva offrire piuttosto un'immagine diversa di Roma, positiva, trionfante e rinnovata, ma, allo stesso tempo, sicura della sua fede e depositaria dell'unica verità, pronta a combattere l'eresia, ma anche madre generosa e comprensiva verso coloro che ritornavano al suo grembo. Decisive, in questo senso, risultavano le figure che circondavano i neoconvertiti, li introducevano nella corte pontificia, quando

2 Per una trattazione più completa di questo tema rinvio ai miei studi “ Roma e gli «ultramontani» : conversioni, viaggi, identità ", Quellen und Forschungen aus italienischen Archiven und Bibliotheken, 81 (2001), p. 351-396 ; "Conversions de voyageurs protestants dans la Rome baroque ”, in R. BABEL und W. PARAVICINI (a cura di), Grand Tour. Adeliges Reisen und europäische Kultur vom 14. bis zum 18. Jahrhundert (Akten der internationalen Kolloquien in der Villa Vigoni 1999 und im Deutschen Historischen Institut, Paris, 2000), Beihefte der Francia 60, Ostfildern, 2004, p. 571-580. 
si trattava di cavalieri, letterati o artisti e nella società romana. Protagonisti furono, in questa funzione di trait d'union, anche eminenti personaggi francesi che gravitavano intorno alla curia pontificia, ma anche artisti più o meno noti, semplici artigiani che nelle loro botteghe raccoglievano connazionali già residenti a Roma, nuovi arrivati in città, ricevevano notizie, fogli volanti - gli Avvisi - libri che la pur vigile censura papale non riusciva sempre a controllare ${ }^{3}$. La presenza francese a Roma non era formata, fra Cinquecento e Seicento solo da pellegrini o rari viaggiatori : era insomma ben radicata in città, come dimostrano le fonti parrocchiali, la chiesa nazionale, le fonti giudiziarie, i documenti curiali che vedevano comunque una presenza all'interno della curia pontificia anche dopo la sua progressiva italianizzazione. Artisti, mercanti, curiali rappresentavano un nucleo solido attorno al quale, poi, si coagulavano presenze transeunti, spesso rappresentate da vagabondi e da persone in cerca di fortuna nelle numerose corti nobiliari e cardinalizie. Sempre più cospicua divenne poi la schiera di diplomatici francesi con un loro seguito, una corte e una familia che proprio nel numero di servitori doveva dare misura del peso politico della figura del sovrano che essi rappresentavano alla corte papale ${ }^{4}$.

Non solo pellegrini, dunque, sebbene la tradizione del pellegrinaggio compiuto soprattutto in occasione degli anni santi avesse ricevuto certamente un nuovo, vigoroso impulso a partire dalla metà del Cinquecento. Era stata una chiara risposta, da parte romana, al diffondersi dell'eresia in Europa e la propaganda cattolica, la curia e la corte pontificia riuscirono a connotare il pellegrinaggio a Roma, alle tombe degli Apostoli, usando anche simboli e linguaggi derivati dalla classicità. Nel tardo Cinquecento, infatti, la classicità utilizzata dalla corte romana non era più quella rinascimentale, ma veniva ora accuratamente selezionata e riutilizzata in funzione della celebrazione del potere pontificio e dell'universalità della chiesa di Roma. Gli esempi potrebbero essere molteplici : basti soltanto citare i trionfi e gli apparati scenici messi in atto in occasioni solenni e le figure della classicità, come ad esempio Costantino, per celebrare proprio Enrico IV tornato, con la sua conversione, in seno alla chiesa romana.

\section{Ercole, Costantino e il re di Navarra}

L'uso funzionale di figure classiche fu infatti messo in atto, in forme esplicite e addirittura clamorose, anche per celebrare la conversione di Enrico di Navarra. Si trattò del messaggio propagandistico accuratamente controllato dall'autorità papale, in contrasto

3 Cf. J. Delumeau, “Contribution à l'histoire des Français à Rome pendant le XVIe siècle ”, Mélanges d'archeologie et d'histoire, 64 (1952), p. 249-286.

4 A questo proposito cfr. O. PONCET, "Les cardinaux protecteurs des couronnes en cour de Rome dans la première moitié du XVIIe siècle : l'exemple de la France ”, in G. SigNOROTTO - M.A. Visceglia (a cura di), La corte di Roma "teatro " della politica europea, Roma, 1998, p. 461-480. 
con la divulgazione delle immagini del re ugonotto che circolavano a Roma prima della sua conversione, immagini riprodotte da incisori francesi e propagandate dal duca di Luxembourg, ambasciatore della Lega cattolica a Roma. La figura mitologica classica alla quale viene associata l'immagine di Enrico di Navarra era Ercole, eroe utilizzato da altri sovrani europei in funzione allegorica, simboleggiando con i suoi attributi sia la forza fisica e il potere militare, che la forza d'animo, la saggezza, la capacità di governare con giustizia ${ }^{5}$. La circolazione delle sue immagini a Roma, prima della riconciliazione con il papa, doveva persuadere che il re francese aveva virtù morali e politiche eccezionali, creare consenso attorno a lui e promuovere l'imitazione delle sue gesta. Queste immagini, a Roma, propagandavano un re eretico e relapso, sebbene già convertito in Francia. L'inquisizione infatti fu presto sulle tracce di chi si era adoperato per la sua diffusione ${ }^{6}$. La colonia francese a Roma, sostenuta dall'ambasciatore della Lega, il duca di Luxembourg, in quell'occasione fu passata al setaccio dal tribunale della fede.

Come è noto, l'abiura di Enrico avvenne il 25 luglio 1593, ma la sua riconciliazione con Roma solo nel 1595, in una cerimonia svoltasi per procura di fronte alla basilica di S. Pietro. In questi due anni però a Roma i circoli francesi si erano assiduamente adoperati in favore del sovrano per legittimarne la sovranità nel cuore del mondo cattolico. Molti personaggi vicini al papa si attivarono presso quest' ultimo per far assolvere il re francese e, fra questi, un peso sicuramente notevole ebbe il cardinale Cesare Baronio, confessore di Clemente VIII. La figura di Baronio, insieme ad altri religiosi appartenenti alla Congregazione dell'Oratorio, fondata da S. Filippo Neri, sarà decisiva per costruire una nuova politica delle conversioni messa in atto fra la fine del Cinquecento e l'inizio del Seicento e che elaborerà una nuova pedagogia della persuasione per convertire gli 'ultramontani' basata proprio su una nuova immagine di Roma e del suo passato. Attorno al cardinal Baronio si era costituito un circolo formato non solo da religiosi oratoriani italiani, ma anche da alcuni gesuiti - ricordiamo che esponenti di spicco dei gesuiti, come Antonio Possevino e Roberto Bellarmino erano stati prima ostili, poi favorevoli alla conversione ed alla pacificazione di Enrico IV - da artisti francesi, come alcuni incisori, stampatori che diffusero le immagini del Navarra, come Philippe Tomassin, Antoine Durafort e i loro apprendisti e collaboratori. C'erano anche predicatori francesi famosi come il padre gesuita L. Richeome, autore di molte opere controversistiche dedicate a Enrico IV e di un Cathéchisme royal, e Séguier. La posizione di spicco degli Oratoriani nel sostenere la causa francese è confermata da

5 F. POLLEROß, "From the exemplum virtutis to Apotheosis: Hercules as an Identification Figure in Portraiture : an Exemple of the Adoption of Classical Forms of Representation ", in A. Ellenius (a cura di), Iconography, Propaganda, and Legitimation, Oxford, 1998, p. 37-62.

6 La trascrizione del processo e un'attenta analisi sono state condotte da R. TEODORI, "Pro Fisco S.ti Offici contra retinentes imaginem pretensi Regis Navarrae ". Tra propaganda e censura. l'immagine di Enrico di Navarra nella Roma di fine Cinquecento, tesi di laurea a.a. 1998-99, Facoltà di Lettere e Filosofia, Università di Roma La Sapienza. 
molteplici testimonianze ma soprattutto riporta in primo piano il ruolo che il fondatore, S. Filippo Neri aveva avuto nel persuadere Clemente VIII ad assolvere il re convertito, a credere alla sincerità della sua conversione e riammetterlo in seno alla chiesa. A Filippo Neri e ai suoi confratelli oratoriani fanno infatti riferimento i mediatori francesi che fra il 1593 e il 1595 si adoperarono per la causa del Navarra e in particolare i cardinali Du Perron, D’Ossat e Nevers. Dunque la circolazione di queste immagini, l'uso di modelli antichi per propagandare il nuovo re di Francia avveniva con il consenso più o meno esplicito di questo circolo oratoriano che si proponeva come interlocutore privilegiato e come mediatore politico fra la Francia e Roma.

Nella città del papa i fautori del re convertito potevano contare su un altro punto di forza che era il capitolo della basilica di S. Giovanni in Laterano che, proprio nel 1593, favorì i negoziati per l'assoluzione del re. Esistevano tra il capitolo e i re di Francia legami di natura economica da quando Luigi XI aveva donato alcuni beni in Linguadoca e Guienna. Le rendite furono sospese nel periodo delle guerre di religione e solo nel 1604 Enrico IV donò al capitolo l'abbazia di Clairac ${ }^{7}$. La riconoscenza del capitolo lateranense si manifestò nel 1608 quando fece erigere da Nicolas Cordier, a proprie spese, la statua bronzea di Enrico IV in veste di imperatore romano, perfettamente conforme alla iconografia regale proposta in Francia negli stessi anni, accompagnata da un'iscrizione che esaltava le virtù del sovrano paragonandolo a Clodoveo. Non si trattava di un monumento funebre, ma di una statua eretta in onore di un sovrano vivo ed era un chiaro messaggio politico del partito francese, ricostituito e compattato a Roma proprio in occasione della conversione e dell'assoluzione di Enrico e che ora usava, per rafforzarsi anche il linguaggio visivo della simbologia del potere derivati dall'antichità. Si può dunque parlare a proposito della celebrazione di Enrico IV del passaggio dall'uso di un simbolo pagano - Ercole - ad uno cristiano, anzi ad un convertito - Costantino - nel quadro di un più generale recupero dell'antico usato politicamente sotto il vigile controllo del potere e della censura pontificia. Il binomio Enrico IV-Costantino diventerà poi un topos del teatro gesuitico del Seicento, dell'iconografia coeva e delle incisioni, destinate a circolare, ormai non più in maniera clandestina, ma per rafforzare il partito francese, il re cristianissimo contro la Spagna e il suo potere in Italia e alla corte papale.

Se il circolo oratoriano era stato decisivo nella questione della legittimazione del re calvinista ed aveva fatto della sua conversione uno strumento di propaganda da consolidare anche con il riuso dei simboli e degli apparati della romanità, della regalità imperiale, un più ampio progetto conversionistico fu messo in atto proprio durante il pontificato di Clemente VIII e si avvalse anche della cultura antiquaria e dell'erudizione portata avanti con successo proprio dai seguaci di S. Filippo Neri. La conversione di

7 Sulle trasformazioni architettoniche della basilica cf. J. FrEIBERG, The Lateran in 1600. Christian Concord in Counter-Reformation Rome, Cambridge, 1995. 
viaggiatori ultramontani assumeva infatti un valore fortemente simbolico e propagandistico per rafforzare l'immagine di Roma e del papato nell'Europa della prima età moderna e la cerimonialità romana diventava, in questo processo, uno strumento di propaganda e di persuasione fondamentale.

\section{Uso dell'antico nelle cerimonie romane}

Le forme della cerimonialità sia sacra che profana si erano modellate sull'idea della renovatio dei fasti della Roma classica ${ }^{8}$. Ma alla metà del Cinquecento, questa idea di continuità viene ad incrinarsi : Roma deve riproporsi come patria comune di fronte alle polemiche scatenate dai protestanti non solo per motivi religiosi, ma anche per cause politiche, legate soprattutto alla identità nazionale gallicana da difendere insieme ai suoi privilegi. Esemplare fu, in questo senso, tutta la polemica antiitaliana e antiromana diffusa in Francia nel Cinquecento, rafforzatasi durante la reggenza di Caterina de' Medici. Gli studi sul libertinaggio erudito hanno mostrato come la polemica contro Roma si faccia in Francia più aspra fra il 1570-1590, proprio gli anni che vedevano, a Roma, la fervida attività di circoli filonavarristi per riconoscere la conversione di Enrico di Borbone. Guillaume Naudé scriveva infatti che « on pardonne à Rome aux athées, aux sodomites, aux libertins et à plusieurs fripons, mais on ne pardonne jamais à ceux qui mesdisent du pape ou de la cour romaine, ou qui semblent révoquer en douce cette toute puissance papale de la quelle les canonistes d'Italie ont tant brouillé de papier ${ }^{9}$ ". $\mathrm{Ma}$, accanto alla polemica e, talvolta connessa con essa in un intreccio difficilmente scindibile, non mancavano lo stupore e l'ammirazione per la città del papa, per la magnificenza delle sue "fabriche ", per lo sfarzo della ritualità che esaltava la maestà del sovrano pontefice e del suo dominio universale.

Le descrizioni delle cerimonie occuperanno infatti gran parte dei resoconti dei viaggiatori francesi a Roma, come Benard che nel 1621 nel suo Voyage dedica un capitolo alla « Description des cérémonies observées quand notre Sainct Père le Pape va par la ville lors qu'il veut aller faire la chapelle et celebrer la saincte messe en quelques Églises dedans ou dehors de Rome, et l'ordre tenu par ceux de sa suite et des chapelles qu'il fait par an 10 " : è la cerimonialità ben regolata che esprime gerarchie e ordine attraverso i colori, gli abiti che colpisce i viaggiatori. Anche alcuni laici, come Étienne Paquier nelle sue lettere non poteva negare che ormai l'antichità era passata e che tutto, a Roma, celebrava la potenza del sovrano pontefice. Scriveva infatti : « Repassez l'an-

8 Sul tema cf. M.A. VISCEGLIA, La città rituale : Roma e le sue cerimonie in età moderna, Roma, 2002.

9 R. PITARD, Le libertinage érudit dans la première moitié du XVIIe siècle, Paris, 1943, p. 262.

10 Le Voyage de Hierusalem et autres lieux de la terre sainte fait par le sieur Benard ensemble son retour par l'Italie, la Suisse, Allemagne, Hollande et Flande, Paris, 1621. 
cienneté, vous trouverez que de tout temps, dépendoit de la chaise de S. Pierre et de ses successeurs en la ville de Rome, l'univers de l'Église générale et universelle ${ }^{11}$. "

La rifondazione della cerimonialità romana, che ora si proponeva come universale e non più cittadina, dopo il Concilio di Trento significò anche la riscrittura della storia antica come storia sacra. Questa operazione si manifestò in forme diverse, alcune delle quali furono immediatamente percepibili dagli occhi dei pellegrini, dei visitatori, e di grande effetto sulla urbanistica cinquecentesca. Si trattava, ad esempio, della ricollocazione degli obelischi davanti alle chiese e alle basiliche : la risacralizzazione di monumenti antichi, con il coronamento della Colonna Traiana con la statua di S. Pietro si propose nel 1587 come un atto fortemente simbolico di Sisto V che, proprio nel suo pontificato, risistemò l'urbanistica romana secondo un ordine definito e funzionale all'esaltazione della potenza e all'universalità del papato tridentino ${ }^{12}$. La risignificazione dello spazio sacro non avveniva solo in superficie e verticalmente, ma, forse in modo più profondo e di forte impatto emotivo, scavando il sottosuolo.

Riscoprire le catacombe, i cimiteri dei martiri, significava elaborare e diffondere nuovi itinerari che ridisegnassero i percorsi delle già diffuse guide di Roma. I mirabilia Urbis degli umanisti, dei letterati cinquecenteschi che esaltavano la suggestione dell'antico dovevano essere riscritti, o sostituiti, con i percorsi della Roma sancta. Questo si doveva offrire ai pellegrini, a chi arrivava a Roma per visitare la città. Anche in questa operazione culturale gli Oratoriani furono protagonisti. Cesare Baronio pubblicava gli Annales ecclesiastici (1588-1607) e il Martyrologium Romanum; Antonio Bosio (15751629) dava alle stampe la Roma sotterranea con minuziosa descrizione delle catacombe e offriva l'immagine di una città nuova, quasi in opposizione speculare alla Roma classica ${ }^{13}$. Gli Oratoriani elaborarono numerosi itineraria, ma soprattutto elaborarono la cultura, l'erudizione, la diffusione di una pedagogia utilizzata nel corso del Seicento per impressionare e convertire chi arrivava a Roma.

Come fu recepita da pellegrini e viaggiatori francesi questa riscrittura della Roma trionfante in chiave religiosa e devozionale, con il riuso dell'antichità fortemente celebrativo del potere papale ? Gérard Labrot ha sostenuto che, a partire dal 1620-1640 : "Ogni viaggiatore è insieme pellegrino e viaggiatore, naturalmente nella misura in cui lo possa e lo voglia 14 . "Infatti, accanto agli stereotipi negativi della sua corruzione, dell'immagine Roma-Babilonia che si trova in gran parte della controversistica, anche francese, sebbene permanga più forte, per ovvie ragioni confessionali, in quella di lingua tedesca, nelle rela-

11 E. PAQUIER, Lettres historiques pour les années 1556-1594, Genève, 1966, lib. X, p. 227.

12 Si veda, a questo proposito, M. FAGIOLO - M.L. MADONNA (a cura di), Sisto V Roma e il Lazio, Roma, 1992.

13 Su questo tema cf. S. DitchfiELD, “ Leggere e vedere Roma come icona culturale (15001800) circa ”, in Roma città del papa, Storia d'Italia, Annali 16, Torino, 2000, p. 31-72. Napoli, 1997, p. 98. 
zioni di viaggio ${ }^{15}$, emerge costante lo stupore e l'attenzione per la Roma moderna, per le opere dei pittori, per le chiese : Grangier de Livernis afferma, visitando S. Pietro, di " être ravi en admiration par ses magnificences, et mes yeux éblouis par ses éclats". Sempre più forte è l'attenzione per palazzi e giardini, per il collezionismo privato : spazi aperti che potevano essere ammirati e permettevano di contemplare la città in completa libertà, come già aveva osservato Montaigne. La Roma moderna, la sua maestosità possono essere ora osservate dall'alto di quei monumenti antichi riutilizzati nella nuova urbanistica : colonne, obelischi non servono per ammirare le rovine, ma per vedere la Roma moderna. Dunque a Roma, da parte della cultura ufficiale, si elabora uno stereotipo di guida tendente a semplificare e valorizzare i luoghi che gli stranieri devono visitare. Sono gli itinerari di Roma cristiana che propongono le catacombe, le chiese dal titolo cardinalizio, la visita alle sette chiese, i rioni, le reliquie dei martiri. Ma i viaggiatori francesi sono attratti dal presente, dalla Roma in superficie, dalle cerimonie che hanno trasfigurato gli antichi trionfi. Nella prima età moderna, fino alla fine del Seicento si può dire che sia la Roma moderna con le sue cerimonie e la magnificenza, denigrata, esempio manifesto della corruzione dei papisti, a prevalere nell'immagine che viaggiatori e pellegrini francesi riportano dal loro incontro con la città eterna. Il fascino delle rovine deve ancora aspettare il XVIII secolo per diventare un topos della cultura europea.

\section{Una Basilica : un simbolo nell'età moderna}

Il contraddittorio fascino che esercitavano alcuni edifici-simbolo della Roma moderna sembra potersi riassumere seguendo le osservazioni di personaggi francesi che fra Seicento e Settecento si trovarono dinanzi a quello che era diventato, da tempo, il simbolo dell'universalità della chiesa di Roma, ma anche del contestato emblema della pretesa supremazia papale. S. Pietro è infatti un nome, un simbolo che assume significati diversi e addirittura opposti nell'età moderna. Immagine-sintesi della cattolicità, della sede del pontefice tornato a Roma dopo la cattività avignonese, dall'inizio del Cinquecento S. Pietro era diventato emblema della corruzione della Chiesa. La costruzione della Basilica aveva drenato i denari delle indulgenze verso Roma, la nuova Babilonia, e Lutero non aveva esitato a scagliarsi nei suoi numerosi scritti, contro il simbolo stesso del papa-anticristo. L'auspicata distruzione del potere papale, con il saccheggio delle sue ricchezze, la dispersione dei suoi simboli, la fuga di chi aveva mercanteggiato nel luogo sacro, sopra la tomba dell'Apostolo, sembrò concretizzarsi al mattino del 6 maggio 1527 quando i lanzichenecchi dettero il sacco alla città. Roma, sconvolta e dissacrata, portava ancora i segni della distruzione quando, nel 1536 fu eletto papa Paolo III Farnese che " fa finalmente di Michelangelo il signore assoluto di un cantiere che non conoscerà più soste. Il penoso spet-

15 Come, ad esempio, N. DE BRALIOn, Seconde partie du premier livre des curiositéz de l'une et de l'autre Rome, Paris, 1658. 
tacolo che veniva offerto dal cuore religioso del mondo è allontanato definitivamente. E nel momento stesso in cui la tomba dell'apostolo fa sorgere un santuario finalmente degno di lei, il successore dell'apostolo dà sistemazione al luogo, così urgentemente necessario nel quale egli, duplice sovrano, si mostrerà, darà udienza... L'opera di Paolo III non si esaurisce con il suo autore. Essa ha sicuramente degli effetti immediati, ben visibili in una città dove le piaghe del Sacco si rimarginano lentamente. Dà prima fiducia e coraggio. Ma è anche gravida del futuro di Roma, poiché, fin dagli inizi, fissa un'ideologia, e priorità d'intervento persistenti e vincolanti ${ }^{16}$ ». Dalla seconda metà del Cinquecento lo spazio antistante la basilica veniva infatti recuperato e segnato dalla romanità e dalla maestà del pontefice che, attraverso le fastose cerimonie, comunicava con un linguaggio politico esplicito e persuasivo, fatto di simboli, colori, precedenze accuratamente studiati e codificati, il trionfo della Chiesa cattolica sull'eresia e la potenza, temporale e spirituale, del vicario di Cristo. Il centro della cattolicità era ormai a S. Pietro : gli interventi architettonici e urbanistici cinquecenteschi avevano infatti spostato definitivamente il baricentro della città. La celebrazione degli Anni Santi, a partire dal Giubileo del 1575, doveva comunicare in maniera esplicita e solenne la maestà e la superiorità della Chiesa di Roma e l'unicità della sua funzione salvifica. Proprio per ridisegnare gli itinerari dei pellegrini, per condurli attraverso un percorso salvifico che celebrasse la maestà della città del papa, Filippo Neri aveva iniziato e diffuso la pia pratica della visita alle Sette Chiese, circuito non solo ideale che disegnava un nuovo itinerario di salvezza nella Città Eterna. Basta osservare l'incisione di A. Lafréry, Le sette chiese di Roma (1575) per comprendere la centralità della basilica Vaticana nel sacro percorso : essa troneggia infatti in primo piano mentre davanti al suo sagrato si inginocchiano i pellegrini, i primi del lungo corteo giunti alla meta.

I significativi interventi urbanistici e architettonici operati da Sisto V nel breve ma intenso pontificato (1585-1590) erano tutti volti a ridisegnare il volto della città per consacrarne definitivamente il suo ruolo di capitale della cattolicità e dello Stato pontificio. Monete e medaglie celebravano la ritrovata quiete, la sicurezza, il consolidato tesoro, pace e abbondanza in un clima di severa moralità controllata dal vigile occhio di papa Peretti. L'iconografia presenta il susseguirsi delle opere architettoniche fra le quali campeggia la facciata di S. Pietro, finalmente terminata e proposta come icona della potenza pontificia. S. Pietro diventa nel Seicento, nell'atmosfera solenne della città barocca, sintesi dei simboli di potere e teatro offerto alla città e al mondo 17 . E'proprio il termine " teatro " a ricorrere con inusitata frequenza negli scritti di Alessandro VII, l'artefice della Roma moderna. Mentre la città combatteva l'epidemia di peste, ai pellegrini si offriva un nuovo, magnifico " teatro ", aperto dal Bernini proprio a partire dal 1656. "Maravigliosi sono etiandio i sontuosi Portici che formano questo Teatro... Questo singolarissimo adornamento, unito alle due meravigliose, e

G. LABROT, Roma “caput mundi ”, op. cit., p. 53.

G. Curcio, Architetture e città. Roma tra 1600 e 1750, in di A. ZuCCARI (a cura di), La storia dei giubilei, III, 1600-17575, Firenze, 1999, p. 138. 
stupende fontane, ed all'Obelisco, non lascia che più desiderarsi ", scriveva quasi un secolo dopo il canonico Giovanni Marangoni nel suo libro Il devoto Pellegrino, diretto a coloro che venivano a visitare le basiliche per l'anno santo 1750 . E ricordava che due erano stati i motivi che avevano mosso papa Chigi a far costruire il " teatro " davanti alla basilica : "il primo, per la grande santità di questo Campo d'intorno e l'altra per la sua grande carità, ed amore Paterno verso i poveri di Roma ", afflitti dalle conseguenze della peste. Ma soprattutto era chiaro il fine politico di questa sontuosa e straordinaria innovazione urbanistica. Scriveva infatti che «il fine più elevato, e sublime fu per accrescere la venerazione a questo campo Vaticano santificato col sangue d'innumerabili Martiri... e questi siti... conveniva che fossero ornati con un sì trionfale ornamento, molto più sontuoso del celebre Campidoglio dell'antica Roma ». Dunque la cupola della basilica e la piazza "si erano sostituiti, nella costruzione dell'immagine di Roma moderna, a quello che un tempo, per i pellegrini che giungevano dall'alto di Monte Mario, era stato il Campidoglio ${ }^{18} »$.

Con la seconda metà del Seicento, con l'intensificarsi della pratica del viaggio in Italia, esperienza educativa per giovani cavalieri, nobili d'Oltralpe, ma anche per sempre più numerosi artisti in cerca di ispirazione nelle tracce del passato, S. Pietro diventa il punto focale della città, il culmine di un itinerario preparato già dalle guide e dalla ormai diffusa letteratura di viaggio, genere assai popolare, capace di forgiare e trasmettere immagini, stereotipi, pregiudizi che entrano a far parte della cultura europea ${ }^{19}$. Con essa, i viaggiatori avevano anche fatto propri i luoghi comuni negativi sul papa, su Roma e i suoi abitanti. Nel 1691, François-Maximilien Misson pubblicava il Nouveau voyage d'Italie, una guida dettagliata delle più importanti tappe del Grand Tour. Oltre a fornire dati precisi utili al viaggiatore, Misson non poteva prescindere dall'aggiungere notazioni personali e aneddoti raccolti durante il suo viaggio. A proposito di San Pietro, Misson osservava che la basilica " passe pour le plus vaste et le plus superbe temple du monde. Pour en bien juger, il faut aller souvent, il faut monter sur les voûtes, et se promener par tout jusque dans la boule qui est sur le dôme ; il faut voir l'Église souterraine. D'abord on ne trouve rien qui paraisse fort étonnant ; la symétrie et les proportions bien observées de l'architecture ont si bien mis chaque chose en son lieu, que cet arrangement laisse l'esprit dans sa tranquillité, mais on se trouve engagé dans la nécessité de l'admirer ". Dopo aver fornito misure e dimensioni della basilica, si sofferma a descrivere la tomba dell'Apostolo, raccogliendo aneddoti che circolavano fra chi faceva da guida ai numerosi, entusiasti viaggiatori. " On descend par un escalier sous cet autel, pour aller à la Chapelle où repose, dit-on, le corps de saint Pierre et pour visiter les autres saints lieux qui sont en divers endroits dans les caves de cette Église. J'ay remarqué à l'entrée de ces grottes une bulle gravée en marbre par laquelle il est défendu aux femmes d'y entrer qu' une seule

19 A. BRILLI, Il viaggio in Italia. Storia di una tradizione culturale, Bologna, 2006. 
fois l'an, à savoir le lundy de la Pentecoste ; et aux hommes de s'y présenter ce jour-là, sous peine d'excommunication contre les uns et contre les autres. Ces lieux sont obscurs ; le sacristain nous a dit qu'une aventure galante avait donné lieu à ce règlement ${ }^{20}$. "Sono parole che lasciano trasparire lo scetticismo a proposito del culto delle reliquie, ma anche la notazione sulla discutibile moralità dei Romani che troppo spesso facevano delle chiese luogo di trasgressioni, anche durante le funzioni sacre.

Le immagini settececentesche di S. Pietro, illuminato dai fuochi di Castel Sant'Angelo per le " allegrezze " che celebravano feste papali, visite di ambasciatori e sovrani, confermano quella dimensione teatrale così fortemente impressa nella mente del viaggiatore europeo, attratto dalle cerimonie e dal fasto della Chiesa cattolica singolarmente in contrasto con la perdita del ruolo politico del papato nell'Europa del tempo. Ma, proprio alla fine del secolo dei lumi, S. Pietro sembrò a molti stranieri, soprattutto ai Francesi, il rifugio sicuro dalla tempesta rivoluzionaria. Nel 1794, Leonard de Tournefort, curato di Ville, in Francia, scriveva un opuscolo in versi dedicato al papa Pio VI, da lui definito così : "modèle de prudence et de piété, il défendit avec zèle les droits de l'Église attaquée, il soutint la foi avec fermeté ; il maintint la discipline et le dogme dans leur pureté, il consola un fidèle clergé, injustement persécuté, il devint le protecteur et père de tous les malheureux 21 ...». Il sacerdote francese, appartenente a quel clero refrattario perseguitato dai rivoluzionari, "chassé plusieurs fois de sa paroisse par des brigands effrenés, arraché et forcé d'abandonner sa patrie, errant dans les bois et obligé de se cacher dans des antres, échappé enfin comme par miracle du milieu de persécutions et des dangers pressants " dichiarava, nel suo opuscolo, di non avere altra ambizione che quella di portarsi nella capitale del mondo cristiano. A Roma, dove era giunto dopo tanto peregrinare, aveva ricevuto consolazione ed era riuscito a non pensare ai disastri subiti dalla sua patria con la contemplazione dei monumenti sacri che Roma offre a ogni passo ». Ma solo uno, simbolo stesso e solenne della Chiesa e del suo pastore, "par sa beauté et magnificence l'emporte sur tous les autres". Solo l'augusta basilica del principe degli apostoli lo aveva consolato e ispirato i suoi versi ; poteva insomma gridare : "Beati qui habitant in domo tua, Domine " mentre in Europa si levavano forti i venti della Rivoluzione.

\begin{tabular}{l} 
Irene FosI \\
\hline Université de Chieti-Pescara \\
Via Antistene 5 \\
I-00124 Roma \\
irenefosi@hotmail.com
\end{tabular}

20 F. M. Misson, Nouveau voyage d'Italie avec un Mémoire contenant des avis utiles à ceux qui voudront faire le mesme voyage, II, Utrecht, 17225, p. 129. Sainteté Pie VI Souverain Pontife, à Rome, 1794, chez A. Fulgoni. 